\title{
Modelagem computacional aplicada ao estudo de um trocador de calor solo-ar com diferentes configurações geométricas
}

\author{
Computational modeling applied to the study of an earth-air heat exchanger with different \\ geometric configurations
}

\author{
B. R. Nunes ${ }^{1}$; J. G. Ferraz ${ }^{1}$; R. Kipper ${ }^{2}$; M. K. Rorigues ${ }^{1}$; R. S. Brum ${ }^{3}$; J. A. \\ Souza $^{1,2}$; L. A. O. Rocha ${ }^{1,3}$; E. D. Dos Santos ${ }^{1,2}$; L. A. Isoldi ${ }^{1,2, *}$ \\ ${ }^{1}$ Programa de Pós-Graduação em Modelagem Computacional (PPGMC),Universidade Federal do Rio Grande \\ (FURG), 96.203-900, Rio Grande-RS, Brasil \\ ${ }^{2}$ Escola de Engenharia (EE), Universidade Federal do Rio Grande (FURG), 96203-900, Rio Grande-RS, Brasil \\ ${ }^{3}$ Programa de Pós-graduação em Engenharia Mecânica (PROMEC), Universidade Federal do Rio Grande do Sul \\ (UFRGS), CEP 90050-170, Porto Alegre-RS, Brasil
}

*liercioisoldi@furg.br

(Recebido em 14 de setembro de 2014; aceito em 29 de dezembro de 2014)

\begin{abstract}
Com o intuito de diminuir o consumo de energia elétrica utilizam-se fontes de energias renováveis, desta forma, é possível aproveitar a radiação solar que atinge a superfície do solo como energia térmica através do uso de trocadores de calor solo-ar (TCSA) em ambientes construídos. Os TCSA são dispositivos onde o ar ambiente externo é resfriado ou aquecido ao circular dentro de dutos horizontalmente enterrados nas camadas superficiais do solo. Esta troca acontece pela inércia térmica do solo, de modo que no verão o ar que circula pelo TCSA sai com temperatura mais baixa que o ar externo; enquanto no inverno o ar externo ganha calor ao circular pelo TCSA, saindo com temperatura mais elevada. Sendo assim, este trabalho tem como objetivo simular numericamente o comportamento térmico e fluidodinâmico de um TCSA, mantendo constante sua área de troca térmica, permitindo a avaliação da influência da configuração geométrica da instalação no potencial térmico do TCSA. Através de um modelo computacional tridimensional, verificado e validado, desenvolvido no software FLUENT (baseado no Método dos Volumes Finitos), obteve-se os resultados numéricos que indicaram uma pequena diminuição no potencial térmico do TCSA em relação à considerável redução da porção de solo necessária para sua instalação, evidenciando a importância e a aplicabilidade da modelagem computacional neste tipo de problema de engenharia.

Palavras-chave: modelagem computacional, trocadores de calor solo-ar, avaliação do potencial térmico
\end{abstract}

In order to reduce the consumption of electricity is used renewable energy sources in this way is it is possible to use the solar radiation that reaches the soil surface as thermal energy by the use of earth-air heat exchangers (EAHE) in built environments. The EAHE are devices in which the external ambient air is cooled or heated when circulating in the horizontally buried ducts. This exchange takes place due to the thermal inertia of the soil, so that in summer the air that circulates into the EAHE exits with a smaller temperature than external air, while during winter the external air gains heat when circulates through the EAHE, leaving it with a higher temperature. Thus, this work aims to simulate numerically the thermal and fluid dynamic behavior of an EAHE, keeping constant its heat exchange area, enabling the assessment of the influence of the geometrical configuration of the installation in the EAHE thermal potential. By means of a tridimensional computational model, verified and validated, developed in the FLUENT software (based on the Finite Volume Method), the numerical results obtained indicated a small reduction in the thermal potential of the EAHE in relation to the considerable reduction of the portion of soil necessary for their installation, highlighting the importance and the applicability of the computational modeling in this kind of engineering problem.

Keywords: computational modeling, earth-air heat exchangers and thermal potential assessment 


\section{INTRODUÇÃO}

O trocador de calor solo-ar (TCSA) é um equipamento onde o ar ambiente é resfriado ou aquecido ao circular dentro de dutos horizontalmente enterrados. Essa troca de calor acontece pela inércia térmica do solo, de modo que, no verão, o ar que escoa pela tubulação tenha queda de temperatura em relação ao ar externo; enquanto no inverno o ar é aquecido ao passar pelo dispositivo, saindo com uma temperatura superior à do ar ambiente. Assim, o ar que sai do duto é direcionado para o interior de edificações, propiciando uma melhoria sua condição térmica e reduzindo significativamente o consumo de energia elétrica dos equipamentos convencionais de condicionamento de ar. No entanto, para os períodos ano entre estas estações o TCSA não apresenta a mesma eficácia e estudos de viabilidade devem ser elaborados para sua operação.

Com base no exposto, a seguir será realizada uma breve revisão da literatura sobre TCSA, mostrando a importância e a aplicabilidade destes dispositivos. No trabalho de Paepe e Janssens [1], foram utilizados métodos analíticos unidimensionais para realizar as análises da eficiência térmica dos TCSA, com relação às suas dimensões, independentemente do tipo de solo e das condições climáticas. Observou-se que o projeto do TCSA depende de três parâmetros: o diâmetro, o comprimento e a quantidade de dutos paralelos. Notou-se também que o desempenho térmico tem uma melhora com a redução do diâmetro do duto e com o aumento do número de dutos enterrados em paralelo. Mais recentemente em Vaz [2], percebeu-se que a necessidade de desenvolver novas tecnologias que viabilizassem o uso de fontes alternativas e sustentáveis de energia era cada vez mais evidente. Dessa forma, foi realizado um estudo experimental sobre a utilização de TCSA no sul do Brasil, na cidade de Viamão. Um modelo computacional tridimensional, foi desenvolvido nos softwares GAMBIT e FLUENT. Os resultados obtidos mostraram a validade e a eficácia do modelo computacional empregado, permitindo a sua utilização para futuras pesquisas e projetos de TCSA.

Em [3], foi desenvolvido um estudo com base no modelo numérico desenvolvido em Vaz [2] a fim de analisar a influência da profundidade da instalação do TCSA em seu desempenho. O modelo computacional de Vaz [2] foi então aprimorado, dando origem ao modelo Simplificado. Os resultados mostraram que a profundidade de instalação dos dutos influencia significativamente o potencial térmico do TCSA. Foi ainda indicada a profundidade de instalação de $3 \mathrm{~m}$ como sendo a ideal entre os casos analisados.

Além disso, em Brum [4] foi apresentado uma nova modelagem computacional para predizer o comportamento térmico dos TCSA. O modelo reduzido exige um menor esforço computacional. Um estudo de caso foi desenvolvido para investigar a influência da profundidade de instalação no potencial térmico de um TCSA. Os resultados estão de acordo com os encontrados na literatura, mas, no entanto, eles foram obtidos com uma redução do tempo de processamento de quase $45 \%$.

Sendo assim, o principal objetivo do presente trabalho é, partindo de um TCSA com configuração geométrica simples, ou seja, um duto reto com $110 \mathrm{~mm}$ de diâmetro instalado a $3 \mathrm{~m}$ de profundidade, propor diferentes configurações geométricas, de maior complexidade, mantendo o mesmo comprimento, o mesmo diâmetro e a mesma profundidade de instalação, porém ocupando uma porção de solo significativamente menor. Tal redução da porção de solo ocupada pelo TCSA pode ser uma vantagem para sua utilização em regiões urbanas, onde os terrenos das edificações possuem dimensões restritas. No entanto é necessário avaliar a influência dessas configurações geométricas complexas para o TCSA em seu potencial térmico.

\section{METODOLOGIA}

Nesta etapa, é apresentada a modelagem matemática que expressa os fenômenos do transporte envolvidos no princípio de funcionamento do TSCA. A equação da energia é o modelo matemático utilizado para avaliar a difusão do calor no solo e a troca térmica entre o ar em escoamento no TCSA e o solo. Para estudar o movimento do ar dentro no duto, serão utilizados os princípios de conservação de massa e de quantidade do movimento. Para analisar o escoamento turbulento, torna-se necessária a modelagem da turbulência. Neste estudo foi 
utilizado o modelo simplificado de Brum et al. [3] e em virtude das geometrias complexas adotou-se o modelo de turbulência $k-\varepsilon$.

\subsection{CONSERVAÇÃO DA MASSA}

A conservação da massa é uma das leis fundamentais da Mecânica Clássica e indica que a massa se conserva. A taxa de acumulação de massa em um volume de controle é igual à massa que entra menos a massa que sai. Na sua forma mais geral, é utilizada para dois tipos de escoamento: escoamentos incompressíveis e escoamentos compressíveis, e é definida por Maliska [5]:

$$
\frac{\partial \rho}{\partial t}+\nabla \cdot(\rho \vec{v})=S_{m}
$$

onde $\rho$ é a massa específica do fluido $\left(\mathrm{kg} / \mathrm{m}^{3}\right), \vec{v}$ é o vetor velocidade do escoamento $(\mathrm{m} / \mathrm{s})$, $S_{m}\left(\mathrm{~kg} / \mathrm{m}^{3} \mathrm{~s}\right)$ é o termo da fonte e $t$ é o tempo (s). Neste estudo, o escoamento é incompressível, $\operatorname{logo}, \partial \rho / \partial t=0$ e, como não existem fontes de massa, $S_{m}=0$. Desta forma, a Eq. (1) pode ser reescrita como:

$$
\nabla \cdot(\vec{v})=0
$$

\subsection{CONSERVAÇÃO DA QUANTIDADE DE MOVIMENTO}

Conforme Maliska [5], a equação da conservação da quantidade de movimento para um sistema não acelerado é dada por:

$$
\frac{\partial}{\partial t}(\rho \vec{v})+\nabla \cdot(\rho \vec{v} \vec{v})=-\nabla \cdot(\tilde{\tau})+\rho \vec{g}+\vec{F}
$$

onde $(\rho \vec{g})$ é a força do campo gravitacional por unidade de volume $\left(\mathrm{N} / \mathrm{m}^{3}\right), \vec{F}$ são as forças do

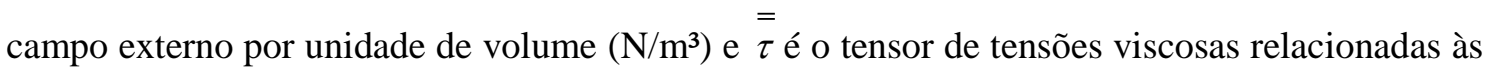
forças de superfície em um elemento infinitesimal de fluido $\left(\mathrm{N} / \mathrm{m}^{2}\right)$. Tendo em vista que o escoamento é incompressível, o tensor de tensões viscosas é representado por:

$$
\bar{\tau}=\mu\left[\left(\nabla \vec{v}+\nabla \vec{v}^{T}\right)\right]
$$

sendo $\mu$ a viscosidade dinâmica do fluido $(\mathrm{kg} / \mathrm{ms})$.

\subsection{CONSERVAÇÃO DA ENERGIA}

De acordo com Maliska [5], a lei da conservação de energia afirma que a quantidade total de energia em um sistema isolado permanece constante. A equação da conservação da energia é dada por:

$$
\frac{\partial \rho h}{\partial t}+\nabla[\vec{v}(\rho h)]=\nabla \cdot\left[k_{e f f} \nabla T\right]
$$

onde $k_{\text {eff }}$ é a soma condutividade térmica adicionada a condutividade térmica turbulenta $(\mathrm{W} / \mathrm{m} \cdot \mathrm{K})$ e $h$ é a entalpia específica $(\mathrm{J} / \mathrm{kg})$, definida por: 


$$
h=\int_{T_{r e f}}^{T} c_{p} d T
$$

sendo $c_{p} \mathrm{o}$ calor específico à pressão constante $(\mathrm{J} / \mathrm{kg} \cdot \mathrm{K})$.

\subsection{MODELO DE TURBULÊNCIA $k-\varepsilon$}

O modelo $k$ - $\varepsilon$ consiste na resolução de duas equações adicionais e é considerado o modelo de turbulência mais utilizado na simulação numérica de escoamentos turbulentos. Sua implementação é simples de ser executada, e o modelo tem sido validado em diversas aplicações mostrando robustez e precisão [6].

Este modelo possui duas equações que representam, respectivamente, a energia cinética turbulenta $(k)$ e a sua dissipação $(\varepsilon)$, sendo o modelo mais difundido e considerado atualmente, como modelo de turbulência padrão para as simulações industriais. Este modelo apresenta soluções rápidas computacionalmente para muitos problemas da engenharia [7]. Cabe destacar que o modelo $k-\varepsilon$ quando usado em TCSA conseguiu reproduzir adequadamente os efeitos da turbulência [8].

\subsection{MODELAGEM NUMÉRICA}

A utilização da simulação numérica para a análise dos escoamentos de fluidos, transferência de calor e demais fenômenos associados é conhecida como Dinâmica dos Fluidos Computacional (CFD - Computational Fluid Dynamics) [7]. Basicamente, uma análise empregando CFD é dividida em três etapas: o pré-processamento, processamento e o pósprocessamento [5].

No pré-processamento, são realizadas a definição do problema físico, a imposição das condições iniciais e de contorno do problema, a definição do domínio computacional e a geração da malha. No processamento, realiza-se a solução do problema. Para isso é necessária a discretização das equações diferenciais representativas do estudo em questão, através de um método de discretização, dentre os quais destacam-se: o Método de Diferenças Finitas; o Método dos Volumes Finitos e o Método dos Elementos Finitos. E, por fim, no pósprocessamento, apresenta-se e analisa-se os resultados. Nesta etapa os pacotes de CFD estão equipados com ferramentas gráficas bastante completas capazes de auxiliar na visualização e interpretação dos resultados.

Neste estudo os softwares utilizados para a modelagem computacional do problema proposto são o GAMBIT, responsável pela etapa de pré-processamento, e o FLUENT, responsável pela etapa de processamento e pós-processamento. Além disso, a planilha eletrônica EXCEL também foi utilizada no pós-processamento dos dados.

O FLUENT é baseado no Método dos Volumes Finitos (MVF). Sabe-se que o objetivo de um método numérico é transformar uma equação diferencial em um sistema de equações algébricas. Assim sendo, as derivadas da função existentes na equação diferencial devem ser substituídas pelos valores discretos da função. Pode-se dizer então que, todo método que para obter as equações aproximadas satisfaz a conservação da propriedade em nível de volumes elementares é um método de volumes finitos [5].

O desenvolvimento do MVF caracteriza-se pela obtenção de equações aproximadas por meio da resolução de balanços de massa, energia e quantidade de movimento em um determinado volume de controle elementar sobre um meio contínuo. A interpretação física direta das equações resultantes da aplicação do método e a possibilidade de aplicá-lo sobre as malhas com espaçamentos não-uniformes, são as duas das principais razões que explicam a aderência ao emprego do método [7].

Cabe destacar ainda que no presente trabalho foi utilizado o esquema de advecção upwind, para os termos advectivos. A direção do escoamento, neste esquema, é levada em consideração para a realização do cálculo do valor de uma determinada variável como, velocidade e 
temperatura na face do volume. Com isso, sempre é garantida a positividade dos coeficientes dos termos advectivos, sendo geradas soluções numéricas ausentes de oscilações numéricas [9].

Além disso, o modelo Coupled foi utilizado para tratar o acoplamento pressão-velocidade neste trabalho, pois possui um desempenho melhor ao ser comparado com algoritmos de solução segregados. Este modelo é indicado para escoamentos transientes quando a qualidade da malha é baixa ou quando são empregados passos de tempos elevados [2].

\subsection{PROCEDIMENTOS NUMÉRICOS}

Os casos analisados numericamente nesse trabalho foram baseados em estudos anteriores apresentados por Vaz [2] e Brum [4]. Assim, considerou-se um solo argiloso como o da cidade de Viamão/RS.

No GAMBIT foram construídos os domínios computacionais dos TCSA que são formados pelo duto enterrado em uma porção de solo. O duto foi representado com um diâmetro de $110 \mathrm{~mm}$ e instalado a uma profundidade de $3 \mathrm{~m}$ [3]. Como hipótese simplificativa o material e a espessura do duto não foram considerados [2]. A discretização do domínio computacional foi feita empregando volumes finitos tetraédricos. Para gerar as malhas utilizou-se o critério para o tamanho da malha de $d / 3$ para o duto e $3 d$ para o solo, sendo $d$ o diâmetro do duto, ou seja, $110 \mathrm{~mm}[10]$.

Ainda, no GAMBIT foram definidos os tipos de condições de contorno para a entrada do duto como velocity inlet (condições do ar na entrada do duto), para a saída do duto como pressure outlet (condição do ar na saída do duto), na superfície superior do solo como wall (variação térmica na superfície) e as demais superfícies do solo foram consideradas isoladas termicamente. Foram ainda identificados os tipos de materiais utilizados no domínio computacional, solid para o solo e fluid para o ar nos dutos. Após esta etapa, as malhas foram geradas e exportadas para o FLUENT.

Para cada uma das simulações apresentadas nesse trabalho considerou-se o intervalo de tempo de 2 anos. O primeiro ano de simulação é necessário para estabilizar a temperatura do solo, e no segundo ano de simulação são feitas as análises necessárias. Utilizou-se um passo de tempo de 3600 s, com um máximo de 200 iterações para cada passo de tempo.

No FLUENT, foram definidas a massa específica $(\rho)$, a condutividade térmica $(k)$ e o calor específico $\left(c_{p}\right)$ do ar e do solo, conforme Tabela 1.

Tabela 1: Propriedade dos materiais

\begin{tabular}{cccc}
\hline & \multicolumn{3}{c}{ Propriedade dos materiais } \\
\cline { 2 - 4 } Componentes & $\rho\left(\mathbf{k g} / \mathbf{m}^{3}\right)$ & $\boldsymbol{k}(\mathbf{W} / \mathbf{m} \cdot \mathbf{K})$ & $\boldsymbol{c}_{p}(\mathbf{J} / \mathbf{k g} \cdot \mathbf{K})$ \\
\hline Solo Argiloso & 1.800 & 2,1 & 1.780 \\
Ar & 1,16 & 0,0242 & 1.010 \\
\hline
\end{tabular}

Na sequência foram definidas as condições de contorno necessárias para a solução do problema: velocidade prescrita do ar na entrada no duto de $3,3 \mathrm{~m} / \mathrm{s}$, temperatura prescrita do ar na entrada do duto em função do tempo, pressão atmosférica prescrita na saída do duto e temperatura prescrita na superfície superior do solo em função do tempo. A variação anual de temperatura na entrada do ar no TCSA $\left({ }^{\circ} \mathrm{C}\right)$ e a variação de anual temperatura da superfície do solo (C) são representadas, respectivamente, por:

$$
\begin{aligned}
& T_{e}(t)=23,18+6,92 \operatorname{sen}\left[\left(1,72 \times 10^{-2} t\right)-26,42\right] \\
& T_{s}(t)=18,70+6,28 \operatorname{sen}\left[\left(1,72 \times 10^{-2} t\right)+26,24\right]
\end{aligned}
$$

Cabe destacar que as Eqs. (7) e (8) são funções periódicas ajustadas estatisticamente a partir de dados experimentais [2]. Essas funções foram inseridas no FLUENT na forma de uma 
Função Definida pelo Usuário (UDF - User Defined Function), que é um algoritmo em linguagem $\mathrm{C}$, que ao ser compilado permite que estas funções sejam impostas como condições de contorno do problema. Para a análise dos resultados, foi monitorada a temperatura de saída do ar do duto, permitindo comparar os resultados com os apresentados em Brum et al. [3], verificando o modelo numérico empregado no presente trabalho.

\section{RESULTADOS E DISCUSSÃO}

Nesta seção serão apresentadas e discutidas as diferentes configurações geométricas para um TCSA, que foram simuladas numericamente através de um modelo computacional tridimensional, permitindo analisar seu princípio de funcionamento, visando avaliar a influência da geometria da instalação no potencial térmico do dispositivo.

Inicialmente, com base no estudo apresentado em Brum et al. [3], o TCSA foi considerado como um duto reto com $26 \mathrm{~m}$ de comprimento (Instalação 1) e, posteriormente, duas instalações com geometrias mais complexas foram numericamente investigadas, mantendo constante a área de troca térmica do TCSA (Instalações 2 e 3).

\subsection{INSTALAÇÃO 1}

A Figura 1 apresenta o domínio computacional da Instalação 1, com suas principais dimensões.

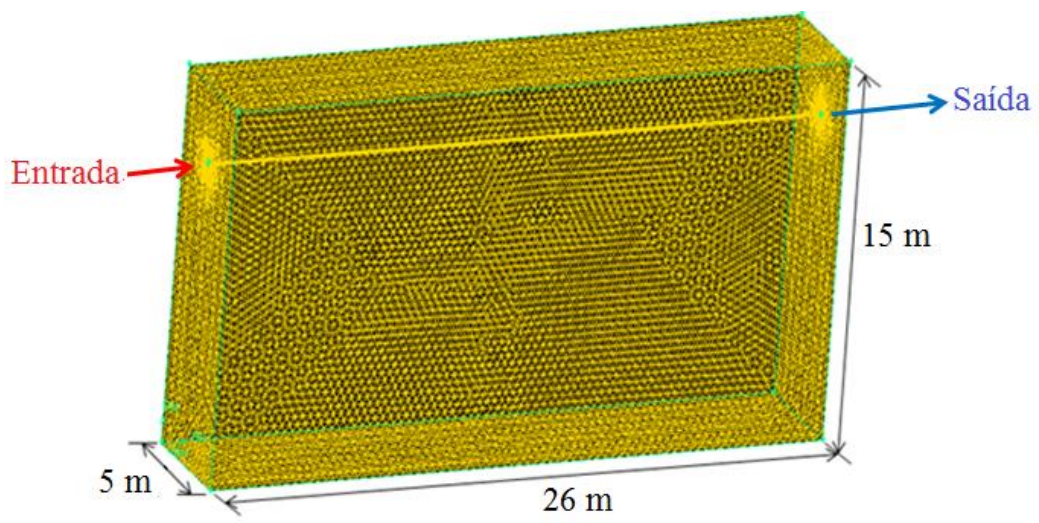

Figura 1: Domínio computacional da Instalação 1

Como dimensões para o solo na Instalação 1, foram usadas $26 \mathrm{~m}$ de comprimento (eixo $x$ ), 15 $\mathrm{m}$ de altura (eixo $y$ ) e $5 \mathrm{~m}$ de largura (eixo $z$ ). Foram geradas as malhas para o solo e para o duto (Figura 1), resultando em 614.581 volumes finitos tetraédricos, sendo 38.729 no duto e 575.852 no solo.

Na Figura 2 é apresentada a variação anual da temperatura na entrada e na saída do TCSA para a Instalação 1. 


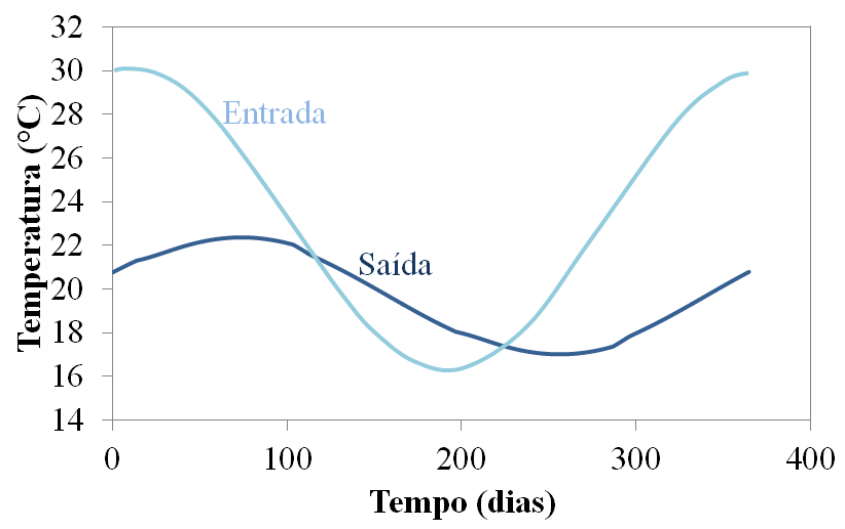

Figura 2: Variação da temperatura do ar na entrada e na saída da Instalação 1

Pela Figura 2 é possível verificar que o TCSA possui um melhor desempenho para resfriamento de ambientes construídos, ou seja, o TCSA é mais efetivo durante períodos de quentes. Nos períodos frios há um aumento na temperatura de saída, porém o TCSA possui uma performance inferior para o aquecimento de ambientes construídos, se comparado com sua performance nos períodos frios. Esse comportamento térmico para o TCSA está de acordo com os resultados previamente apresentados em Brum et al. [3] e Brum [4].

\subsection{INSTALAÇÃO 2}

Para a construção dessa geometria foi respeitado um espaçamento mínimo entre trechos paralelos do TCSA de $1 \mathrm{~m}$. Essa distância mínima é usada para evitar que a troca térmica que existe entre um trecho do duto e o solo interfira de maneira significativa na troca térmica entre outro trecho de duto e o solo, de acordo com os estudos apresentados em Kipper [8]. Assim, na Figura 3 é apresentado o domínio computacional da Instalação 2, indicando suas principais dimensões.

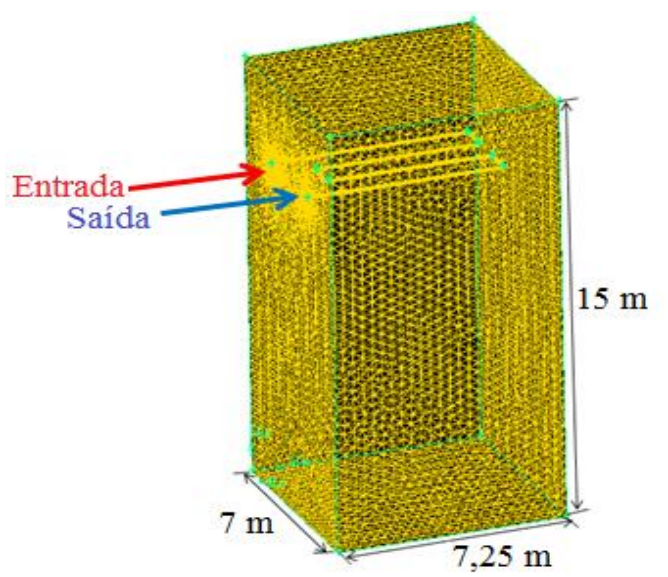

Figura 3: Domínio computacional da Instalação 2

$\mathrm{Na}$ Instalação 2 a porção de solo adotada possuis 7,25 m de comprimento (eixo $x$ ), $15 \mathrm{~m}$ de altura (eixo y) e $7 \mathrm{~m}$ de largura (eixo $z$ ). Foram geradas as malhas, conforme descrição anterior, para o duto e para o solo (Figura 3), resultando em 350.803 volumes finitos tetraédricos, sendo 44.702 no duto e 306.101 no solo.

A solução transiente para a variação de temperatura na saída do TCSA da Instalação 2 é mostrada na Figura 4. 


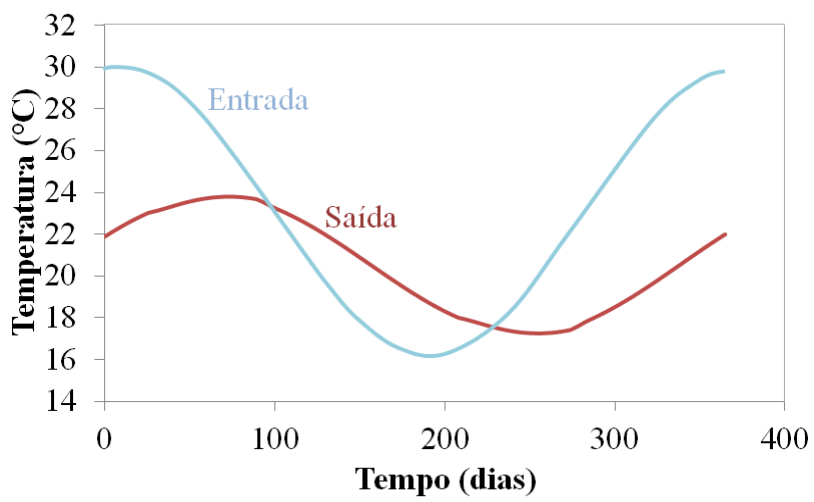

Figura 4: Variação da temperatura do ar na entrada e na saída da Instalação 2

É possível observar na Figura 4 a mesma tendência para o comportamento térmica do TCSA da Instalação 2 se comparado ao da Instalação 1, apesar da significativa redução da porção de solo da Instalação 2.

\subsection{INSTALAÇÃO 3}

Para a Instalação 3 foi considerada uma porção de solo com $7 \mathrm{~m}$ de comprimento (eixo $x$ ), 15 $\mathrm{m}$ de altura (eixo y) e $6 \mathrm{~m}$ de largura (eixo $z$ ), na qual o TCSA foi instalado com a configuração geométrica indicada no domínio computacional apresentado na Figura 5. Assim como na Instalação 2, o espaçamento mínimo de $1 \mathrm{~m}$ entre dutos paralelos foi respeitado.

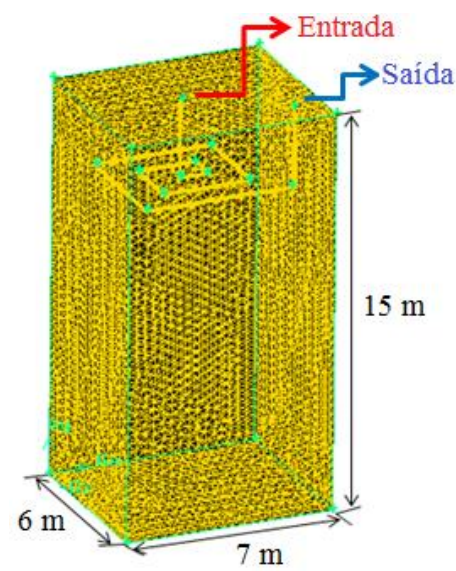

Figura 5: Domínio computacional da Instalação 3

O domínio computacional da Instalação 3 foi discretizado, de acordo com o procedimento descrito anteriormente, com um total de 371.238 volumes finitos tetraédricos, sendo 55.458 no duto e 315.780 no solo (ver Figura 5).

Na Figura 6 é então apresentada a variação da temperatura na entrada e na saída do TCSA em função do tempo para a Instalação 3. 


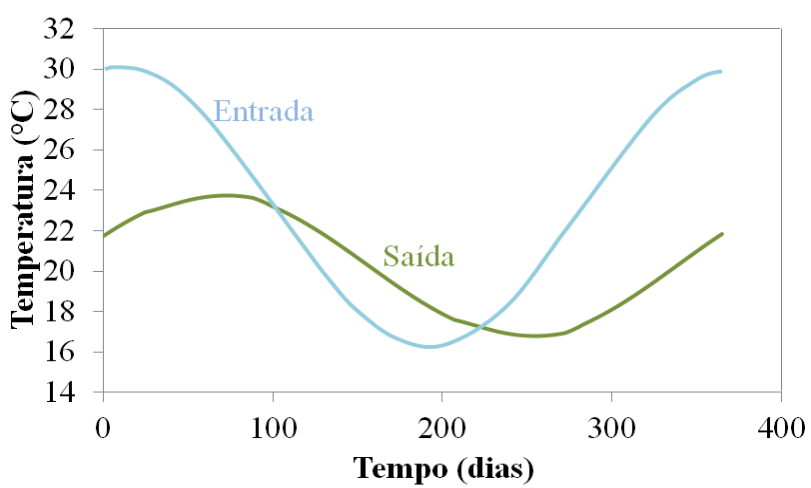

Figura 6: Variação da temperatura do ar na entrada e na saída da Instalação 2

Assim como aconteceu na Instalação 2, qualitativamente, temos um comportamento térmico bastante semelhante da Instalação 3 se comparado à Instalação de referência que é a Instalação 1, mesmo com a redução da quantidade de solo necessária para a instalação do dispositivo.

A seguir uma comparação entre as instalações será realizada, possibilitando discutir quantitativamente as diferenças encontradas.

\subsection{COMPARAÇÃO ENTRE AS INSTALAÇÕES}

Como foi observado nas Figs. 2, 4 e 6 as Instalações 1, 2 e 3 possuem um comportamento térmico semelhante, conforme pode ser comprovado na Figura 7.

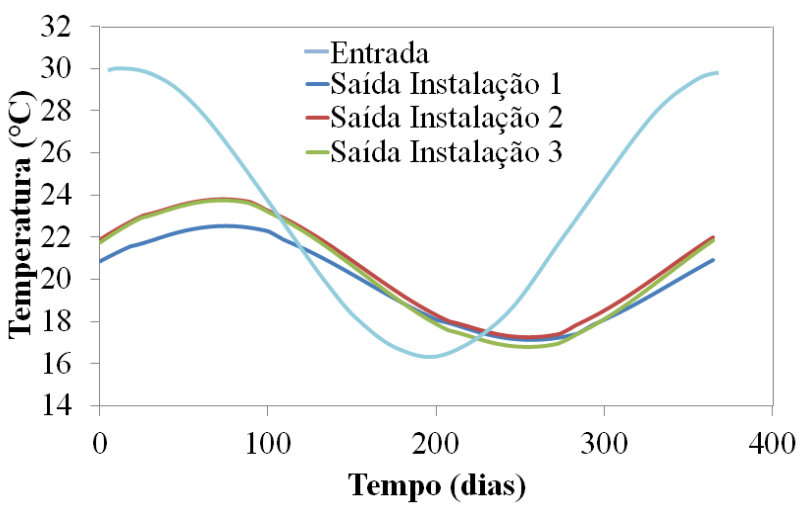

Figura 7: Variação da temperatura do ar na entrada e na saída das Instalações 1, 2 e 3

É possível perceber pela Figura 7 que as Instalações 2 e 3 apresentaram um comportamento térmico inferior, tendo uma redução na variação máxima da temperatura de $1^{\circ} \mathrm{C}$, aproximadamente, em relação à Instalação 1. Entretanto existiu uma redução no volume da porção de solo de 60,9 \% e de 67,6 \%, respectivamente, nas Instalação 2 e 3 quando comparadas à Instalação 1 que serviu como referência nesse estudo.

Sendo assim, essa redução significativa no volume ocupado pelo TCSA justifica a pequena diferença existente no comportamento térmico entre as instalações estudadas. Destacando que a instalação de um TCSA em porções de solo com dimensões reduzidas permite a utilização desses equipamentos e zonas residenciais onde as dimensões dos terrenos são relativamente pequenas. 


\section{CONCLUSÃO}

Neste trabalho foi realizado um estudo numérico do comportamento térmico de um TCSA com diferentes configurações geométricas, visando identificar a influência da geometria da instalação no potencial térmico do equipamento.

Os resultados numéricos mostraram que as instalações com geometrias mais complexas, chamadas no trabalho de Instalações 2 e 3, apresentaram um comportamento térmico ligeiramente inferior, tendo uma redução na variação máxima da temperatura em torno de $1{ }^{\circ} \mathrm{C}$, em relação à instalação de referência (Instalação 1, onde o TCSA possui um duto reto). No entanto, a redução da porção do solo necessária para as Instalações 2 e 3 foi significativa, sendo de aproximadamente $60 \%$ e $70 \%$, respectivamente, em comparação ao volume de solo da Instalação 1. Portanto, pode-se concluir que, do ponto de vista térmico, é possível empregar configurações geométricas de maior complexidade para os TCSA, diminuindo a área ocupada para a sua instalação, viabilizando a utilização desses dispositivos em zonas urbanas, sem que ocorra uma diminuição considerável no desempenho do equipamento.

Como proposta de continuidade deste trabalho, é sugerida a investigação de outras configurações geométricas para os TCSA, especialmente as que tenham um formato tridimensional (por exemplo, um helicoide). É importante também avaliar a perda de carga causada pela complexidade geométrica das instalações.

\section{AGRADECIMENTOS}

À Universidade Federal do Rio Grande (FURG) e à Coordenação de Aperfeiçoamento de Pessoal de Nível Superior (CAPES) e ao Conselho Nacional de Desenvolvimento Científico e Tecnológico $(\mathrm{CNPq})$ por todo auxílio prestado para a realização do presente trabalho.

\section{REFERÊNCIAS BIBLIOGRÁFICAS}

1. Paepe MD, Janssens AA. Thermo-hydraulic design of earth-air heat exchangers. Energ Buildings. 2003 May;35(4):389-97.

2. Vaz J. Estudo experimental e numérico sobre o uso do solo como reservatório de energia para o aquecimento e resfriamento de ambientes edificados [thesis]. Porto Alegre (RS): Universidade Federal do Rio Grande do Sul (UFRGS); 2011. 235 p.

3. Brum R, Rocha LAO, Vaz J, Dos Santos ED, Isoldi LA. Development of simplified numerical model for evaluation of the influence of soil-air heat exchanger installation depth over its thermal potential. International Journal of Advanced Renewable Energy Research. 2012 Dec;1(9):505-14.

4. Brum R. Modelagem computacional de trocadores de calor solo-ar [dissertation]. Rio Grande (RS): Universidade Federal do Rio Grande (FURG); 2013. 120 p.

5. Maliska CR. Transferência de calor e mecânica dos fluidos computacionais. Rio de Janeiro: LTC; 2010. 433 p.

6. Brasil Jr CPA. Turbulência. Rio de Janeiro, 2002.

7. Versteeg HK, Malalasekera W. An Introduction to Computational Fluid Dynamics: The Finite Volume Method. New York: Pearson-Prentice Hall; 2007. 503 p.

8. Kipper R. Análise Numérica de trocadores de calor solo-ar para diferentes configurações de instalação [monograph]. Rio Grande (RS): Universidade Federal do Rio Grande (FURG); 2014. 44 p.

9. Dos Santos ED. Análise numérica de escoamentos turbulentos não reativos com transferência de calor por convecção e radiação térmica em meios participantes [thesis]. Porto Alegre (RS): Universidade Federal do Rio Grande do Sul (UFRGS); 2011. 188 p.

10. Rodrigues MK. Modelagem computacional aplicada à melhoria do desempenho térmico do trocador de calor solo-ar através do método Constructal Design [dissertation]. Rio Grande (RS): Universidade Federal do Rio Grande (FURG); 2014. 151 p. 